\title{
Privacy-preserving association rule mining for horizontally partitioned healthcare data: a case study on the heart diseases
}

\author{
NIKUNJ DOMADIYA* and UDAI PRATAP RAO \\ Sardar Vallabhbhai National Institute of Technology, Surat 395007, India \\ e-mail: domadiyanikunj002@gmail.com
}

MS received 1 October 2016; revised 4 October 2017; accepted 6 February 2018; published online 30 June 2018

\begin{abstract}
In recent years, a trend of electronic health record (EHR) system can be seen increasingly in the hospitals, which has generated huge amount of electronically stored data of patients. Association rule mining technique is very helpful in the numerous applications of healthcare (e.g., correlation between disease and symptoms, disease and offering effective treatment and predicting risks of disease based on the historical data, etc.). The data collected by an EHR system are very important for the medical research. Currently, a patient health report is derived on the basis of a physician's own experience and on the association rule mining results of a local EHR system maintained by a particular hospital. Association rule mining results will be more accurate if the data of all local EHR systems are integrated and association rule mining is performed. Integration of local EHR systems requires the sharing of local EHR data. Sharing of patient records violates the privacy of patients. Hence, medical research is focused on the problem of mining association rules without sharing of local private EHR data. Privacy-preserving distributed association rule mining (PPDARM) solves this issue by mining the association rules while preserving the privacy of patients. In this paper, an approach for the PPDARM is proposed for collaboratively performing association rule mining by all local EHR systems while preserving the privacy. The proposed approach is also analysed with the heart disease dataset.
\end{abstract}

Keywords. Association rule mining; privacy; secure multiparty computation; privacy-preserving data mining; healthcare; heart disease.

\section{Introduction}

Digitalization of healthcare with an electronic health record (EHR) system [1] can store a huge amount of data. Data collected by an EHR system can be used for the medical research and for the diagnosis of diseases. Data mining techniques, such as association rule mining, have received a great deal of attention of medical researchers for predicting the disease or offering effective treatment based on the patient's symptoms [2]. Currently, association rule mining technique uses the data of only local EHR system. Hence, the accuracy of results is lower. Association rule mining on local EHR data is used for discovering the relation between various diseases (heart disease, cancer, Chronic Obstructive Pulmonary Disease (COPD), etc.) and patient attributes [3-5].

The accuracy of association rule mining can be increased, if one integrates all local EHR data to some central server. Association rule mining on data at the central server gives a better accuracy compared with the results of local EHR data. Privacy of information collected by local EHR system is necessary because of the significant

*For correspondence psychological, economic and social harm that can come to an individual when the personal health information is disclosed $[6,7]$. For a high quality medical research for analysing the correlation of patient and attributes, the data from different local EHR systems are required and the privacy of individual patient must be maintained at the same time.

Human life has been affected by life-threatening diseases. Heart disease has received a major focus of the medical researchers compared with any other life-threatening diseases. Australian Bureau of Statistics and Cancer Biology $[8,9]$ investigate the heart disease and cancer as the major causes of death in UK and Australia as shown in figure 1.

Diagnostic systems of heart diseases are costly, time consuming and prone to error. A heart disease patient must be kept under observation as any inappropriate treatment can be fatal. Proper identification of a disease and an early treatment are essential for curing life-threatening diseases. The potential of data mining has been identified by World Health Organization (WHO) for medical research [10] early in 1997. Research at WHO had focused on extracting useful knowledge from huge medical data available from different EHR systems. It could be helpful for the disease prevention, medical diagnosis and treatment, patient health analysis and 

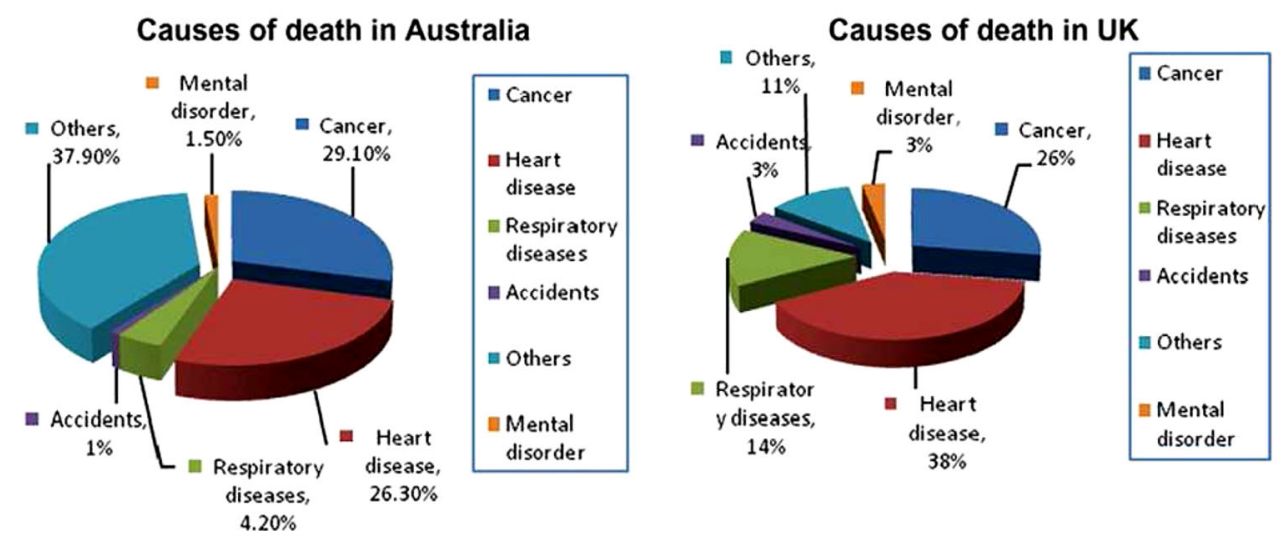

Figure 1. The ratio of different causes of death in Australia (2009) and UK (2006) $[8,9]$.

healthcare service management. Based on these views and issues, this research has focused on mining association rules from the distributed healthcare data for the diagnosis of heart disease.

In this paper, an approach called privacy-preserving distributed association rule mining (PPDARM) for healthcare data is proposed. This approach preserves the privacy of an individual patient and generates accurate results. Heart disease dataset [11] publically available on UCI repository is used for analysing the proposed approach.

\subsection{Our contributions}

Our contributions to the current research are summarized as follows:

1. Initially, we investigate how the accuracy of medical research can be increased by the collaborative association rule mining and at the same time, privacy issues need to be focused.

2. We propose an efficient approach (PPDARM for medical research) on horizontally partitioned data for higher accurate association results and preserving the privacy of patients.

3. We analyse the proposed scheme with heart disease dataset available publically on UCI repository. The analysis of results shows that the accuracy of association among the diseases and symptoms increases by the collaborative mining and privacy of patients is preserved.

The structure of this paper is as follows. Section 2 presents the different concepts related to the heart disease, data partition model and distributed association rule mining. Section 3 presents the proposed PPDARM approach. Section 4 illustrates dataset details and results. Finally, section 5 concludes the paper with some future research directions.

\section{Related concepts}

This section gives a brief overview of heart disease, data partition model and distributed association rule mining.

\subsection{The heart diseases}

This section discusses the characteristics of heart disease, symptoms and causes.

2.1a Characteristics of the heart disease: Medical researchers have analysed the history of heart disease [12]. The analysis of heart diseases by the medical researchers identifies different conditions in which the heart does not function properly [13]. It can cause serious fatal problems to human health. The major types of heart disease are [12]: coronary, congenital, angina, atherosclerosis, rheumatic, arrhythmia and myocarditis.

2.1b Symptoms of the heart disease: The symptoms of heart disease vary from patient to patient. However, in majority of the cases, symptoms are not identified earlier and the disease is identified in an advanced stage. The general symptoms of heart disease are

- pain in chest (angina pectoris),

- discomforts in chest area,

- palpitations,

- shortness of breath, dizziness, headedness, sweating,

- irregular heartbeat and

- fluid retention.

2.1c Attributes of the heart disease: Attributes related to heart disease are not very much clear. However, major attributes such as gender, age, patient history and locality are considered for different knowledge extractions. Other attributes such as high cholesterol, hypertension, foods, obesity, lack of exercise, high blood pressure, stress and diabetes also increase the prospects of heart disease [4, 5]. 


\subsection{Data partition model}

In distributed environment, two major types of data partition model among all participants (hospital with local EHR systems), viz. horizontally partitioned and vertically partitioned data model are given. Here, we discuss existing approaches towards privacy-persevering distributed association rule mining of both kinds of data partitioning.

In horizontally partitioned data, all participants have the same schema, but each participant contains the records of different entities. As shown in figure 2, all local EHR systems have the same schema but each local EHR system contains the data of different patients. Some existing solutions for privacy-preserving association rule mining with horizontal partition data are presented in [14-25].

In case of heart disease research at the global level, many private hospitals with their local EHR system data want to undertake the association rule mining. Each local EHR system contains the data about patients' ID, sex, age, disease, treatment, duration, etc. These hospitals are interested in a collaborative research for finding the relation among sex, age, diagnosis and disease by the global dataset. As a result of collaborative association rule mining, all the hospitals learn association rules, for example, $\{$ restecg $=$ hypertrophy and type of chest pain $=$ asymptomatic and slope $=$ flat and thal $=$ reversible $\}=$ $>\{$ Heart Disease chance $=$ Yes $\}$ is true at the global level. However, to ensure the privacy of patient (as per some privacy act) and confidentiality of local patterns, the hospitals would not want to share their local EHR system data. Hence, the PPDARM on horizontally partitioned data has received lot of attention of the medical research.

In case of vertically partitioned data, each participant has different schema and it stores the data of the same set of entities. Privacy-preserving association rule mining on vertically partitioned data is discussed in [26-31]. As shown in figure 3, the hospital EHR system stores the data of patients and the cell-phone company stores the call details of these patients based on common ID.

In case of medical research for identifying the effect of a cell phone on a particular disease, hospital and cellphone company want to undertake the association rule mining. As a result of this collaborative research, hospital learns the association rule, for example, people with mobile usage of $18 \mathrm{~h} /$ week have a high risk of brain tumour However, hospital and cell-phone company could not reveal the data of cell-phone user and patient, respectively. Hence, PPDARM on vertically partitioned data gives collaborative results and maintains the privacy of users.

In this paper, collaborative data mining on horizontally partitioned data has been focused as major hospitals with EHR systems have the same schema and store the information of different patients. Next, association rule mining on horizontally partitioned data has been discussed.

\subsection{Distributed association rule mining}

Distributed association rule mining [32] can be defined as follows: let $I=\left\{i_{1}, \ldots i_{m}\right\}$ be a set of distinct itemsets present in a dataset $D$. The dataset $D$ is horizontally partitioned in such a way that $D=\left\{D_{1} \cup D_{2} \cup D_{3} \ldots D_{n}\right\}$, $D_{i} \cap D_{j}=\emptyset, 1 \leq i \neq j \leq n$. Association rule is defined as $X \rightarrow Y$, where $Y \subset I, X \subset I$ and $Y \cap X=\emptyset . X$ is called left hand side (LHS) and $Y$ is called right hand side (RHS) of the rule. Association rule analyses the probabilities of RHS set whenever LHS set occurs. The usefulness and interest measure of association rule are represented by support and confidence of the rule.

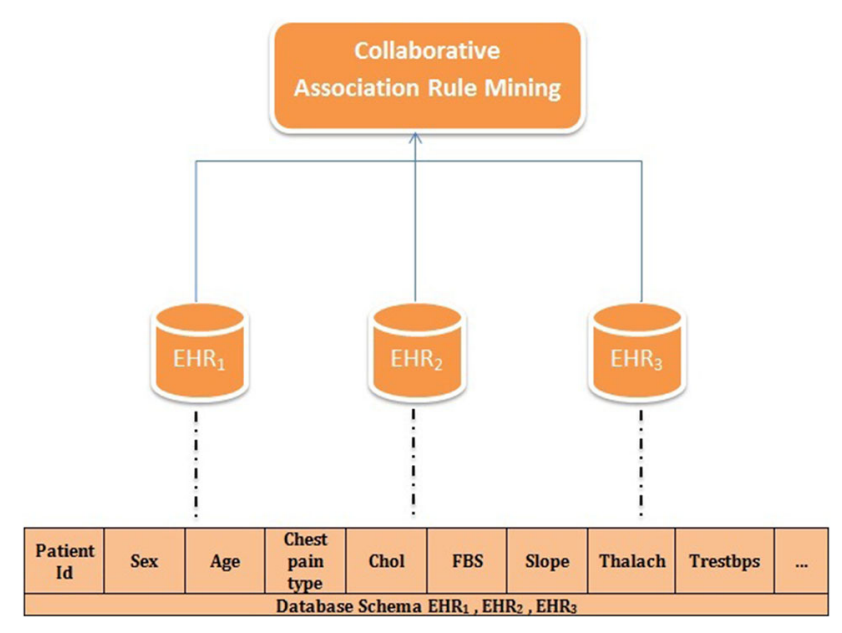

Figure 2. Horizontal partition data model.

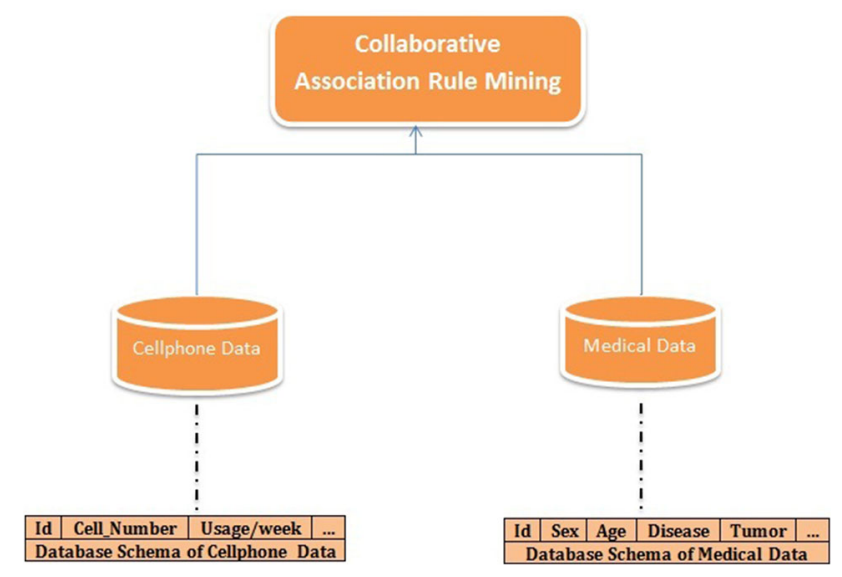

Figure 3. Vertical partition data model. 


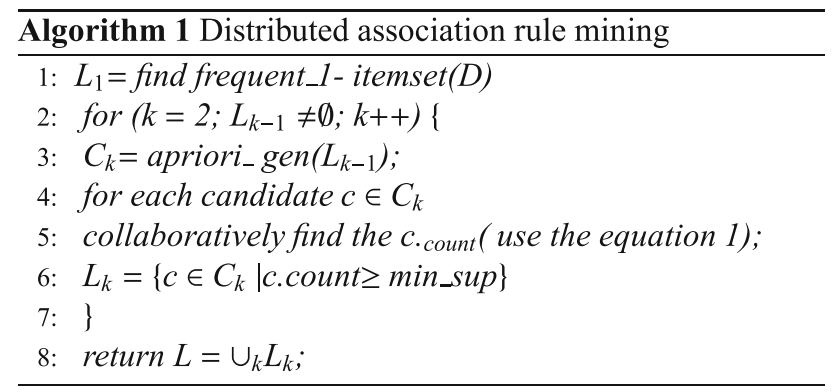

In horizontally partitioned data, support and confidence of the rule (XYZ) are calculated using the following equations:

$$
\operatorname{support}(X Y Z)=\frac{\sum_{i=1}^{\text {no. of participants }} \text { support_count_XYZ }(i)}{\sum_{i=1}^{\text {no. of participants }} \text { database_size }(i)},
$$

$$
\operatorname{support}(X Y)=\frac{\sum_{i=1}^{\text {no. of participants }} \text { support_count_XY }(i)}{\sum_{i=1}^{\text {no. of participants }} \text { database_size }(i)},
$$

$$
\text { confidence }(X Y \rightarrow Z)=\frac{\operatorname{support}(X Y Z)}{\operatorname{support}(X Y)}
$$

Association rule mining is a well-known pattern discovery technique. It has been used in many areas, including medical research [2-5, 7, 33-35]. Apriori is a widely used algorithm among different algorithms for association rule mining. The popular apriori algorithm for horizontally partitioned data is shown in algorithm 1 [32]. This algorithm starts with finding 1 -frequent itemsets $\left(L_{1}\right)$ from the original dataset $D$. Then it finds the set of candidate $k$ itemsets $\left(C_{k}\right)$ base on previously calculated set of frequent $(k-1)$-itemsets $\left(L_{k-1}\right)$. The itemsets of candidate set $\left(C_{k}\right)$ whose count is greater than or equal to minimum support are added to the set of frequent k-itemsets $\left(L_{k}\right)$. In this paper, the apriori algorithm for the association rule mining is used.

\section{Privacy-preserving collaborative association rule mining for the medical research}

\subsection{Problem definition}

In all, $n$ participants (hospitals with the EHR system) $\left\{P_{1}, P_{2}, P_{3}, \ldots, P_{n}\right\}$ with horizontally partitioned data $\left\{\mathrm{EHR}_{1}, \mathrm{EHR}_{2}, \mathrm{EHR}_{3}, \ldots, \mathrm{EHR}_{n}\right\}$ collaborating for medical research have been considered. Each participant $P_{i}$ has privately known data $x_{i}$ from $Z_{p}$. All participants are interested to find $\sum_{i=0}^{n} x_{i}$, while preserving the privacy of an individual. Hence, the goal of all participants is to calculate the following function without disclosing the individual private value $x_{i}$ :

$$
f(x)=\sum_{i=0}^{n} \mathbf{x}_{i}
$$

In the proposed approach, participants are assumed to be semi-honest or passive adversarial and curious [36]. That is, all participants follow the algorithm and provide the correct data. The curious participants try to infer the private value of others by manipulating the received data. Further, our approach requires a minimum of three participants in the collaboration.

It is assumed that the communication channels among the participants are insecure. Any adversary can cache all communications among the participants to learn the private value of some other participants. To solve this issue, the concept of $\mathrm{CDH}$ (Computational Diffie-Hellman [37]) is used. It is computationally secure [38].

The proposed approach is secure based on the assumption that it is computationally hard to solve the $\mathrm{CDH}$ problem.

\subsection{Definition and notations}

Selection of groups $G_{1}$ and $G_{2}$ are as follows: two large prime numbers $p$ and $q$ of the same length are chosen in such a way that $q$ divides $p-1$. A cyclic multiplicative group $G_{1}$ of $q$-order is defined as $<g_{1}>$, where the generator $g_{1}$ and a random number $h \in Z p$ are selected as

$$
g_{1}=h^{(p-1) / q} \bmod p \quad\left(g_{1} \neq 1 \bmod p\right) .
$$

Similarly, the cyclic multiplicative group $G_{2}$ of $q$-order is defined as $\left\langle g_{2}\right\rangle$ where generator

$$
g_{2}=g_{1}^{p} \bmod p^{2}
$$

Definition 1 (CDH problem): Given only $g, g^{a}, g^{b} \in G$ where $a, b \in Z$, computing $g^{a b}$ without knowing $a$ and $b$ is computationally intractable, where $G$ is a multiplicative group with generator $g$.

Definition 2 (DDH problem): Decisional Deffei-Hellman (DDH) problem can be defined as follows: given $g, g^{a}, g^{b}, g^{c} \in G$ where $a, b, c \in Z$, deciding $g^{a b}=g^{c}$ is computationally intractable.

We use the modular property exploit by Taeho et al [38] for privacy-preserving sum in our approach as shown in Eq. (5):

$$
(1+p)^{m}=\sum_{i=0}^{m}\left(\begin{array}{c}
m \\
i
\end{array}\right) p^{i}=1+m p \bmod p^{2} .
$$

Using Eq. (5), multiplication is converted to addition as follows: 


$$
\prod_{i=1}^{n}(1+p)^{x_{i}}=\prod_{i=1}^{n}\left(1+p x_{i}\right)=\left(1+p \sum_{i=1}^{n} x_{i}\right) \bmod p^{2} .
$$

\subsection{Proposed approach for distributed privacy- preserving association rule mining}

In this section, the proposed approach is presented for distributed association rule mining while preserving the privacy of an individual participant's data. Further, the approach is shown to be secure against passive adversaries.

Next, how the $n$ participants compute $C_{\text {count }}=\sum_{i=1}^{n} C_{\text {count }(i)}$ without disclosing the private value $C_{\text {count }(i)} \in G_{1}$ is discussed. The basic idea is to find a random number $R_{i} \in G_{1}$ in such a way that $\sum_{i=1}^{n} R_{i}=0 \bmod p$.

The proposed approach of privacy-preserving computation of $C_{\text {count }}$ is performed in three phases as shown in algorithm 2 .

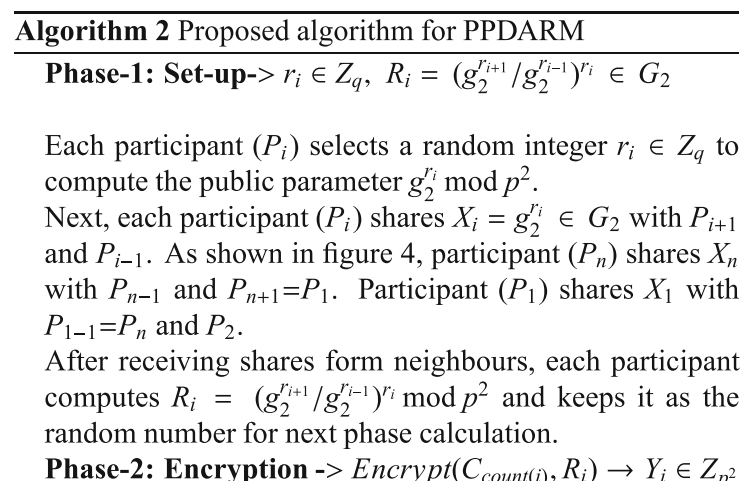

At the end of phase-1, each participant $P_{i}$ has calculated a secret random number $R_{i}$. Ciphertext $Y_{i}$ is calculated using the random number $R_{i}$ by each participant $P_{i}$ as follows:

$$
Y_{i}=\left(1+C_{\text {count }(i)} p\right) R_{i} \bmod p^{2} .
$$

Next, each participant broadcasts the ciphertext to all other participants as shown in figure 5 .

Phase-3: $\operatorname{Sum} \operatorname{Sum}\left(Y_{1}, Y_{2}, Y_{3}, \ldots . Y_{n}\right) \rightarrow \sum_{i=1}^{n} C_{\text {count }(i)} \in$ $Z_{p}$

Once each participant receives the cipher text from all other participants, calculate the final sum as follows:

$$
\begin{aligned}
Y & =\prod_{i=1}^{n} Y_{i} \bmod p^{2} \\
& =\prod_{i=1}^{n}\left(1+C_{\text {count }(i)} p\right)\left(\left(_{g_{2}^{i+1}}^{r_{i+1}} / g_{2}^{r_{i-1}}\right)^{r_{i}}\right) \bmod p^{2} \\
& =\left(1+p \sum_{i=1}^{n} C_{\text {count }(i)}\right) g_{2}^{\sum_{i=1}^{n} r_{i+1} r_{i}-r_{i} r_{i-1}} \bmod p^{2} \\
& =\left(1+p \sum_{i=1}^{n} C_{\text {count }(i)}\right) \bmod p^{2} .
\end{aligned}
$$

The final $C_{\text {count }}$ value calculated by each participant is $C_{\text {count }}=\sum_{i=1}^{n} C_{\text {count }(i)}=(Y-1) / p$.

\subsection{Security analysis}

Since the communication channel is insecure in our approach, any adversary can capture all communications among the participants and can try to infer the private value of some participants. Hence, the security of proposed approach against an adversary is discussed.

Claim: Our approach is $\mathrm{CDH}$ secure in $G_{2}$

Proof To infer any participant's private value, an adversary has ciphertext as $Y_{i}=\left(1+C_{\text {count }(i)} p\right) R_{i} \bmod p^{2}$. Adversary has to solve the random number $R_{i}=\left(g_{2}^{r_{i+1}} / g_{2}^{r_{i-1}}\right)^{r_{i}}$. Here, any adversary has only values $X_{i-1}=g_{2}^{r_{i-1}}, X_{i}=g_{2}^{r_{i}}$ and $X_{i+1}=g_{2}^{r_{i+1}}$ by an insecure communication channel. Next, an adversary can find $X_{i+1} / X_{i-1}=g_{2}^{r_{i+1}-r_{i-1}}$. Now, if any adversary can solve $g_{2}^{\left(r_{i+1}-r_{i-1}\right) r_{i}}$, then that adversary can solve the CDH problem defined in the group $G_{2}$. However, the CDH problem is proved to be hard in the finite field $\mathrm{F}_{p^{2}}$. Hence, any adversary cannot solve $g_{2}^{\left(r_{i+1}-r_{i-1}\right) r_{i}}$ in $G_{2}$.

Therefore, inferring any participant's private value $\left(C_{\text {count }_{(i)}}\right)$ by any adversary is as hard as solving the $\mathrm{CDH}$ problem in $G_{2}$. Hence, the proposed approach is $\mathrm{CDH}$ secure in $G_{2}$.

\section{Performance evaluation}

In this section, theoretical and practical analysis of the proposed approach is discussed. Computation and communication complexities of the proposed approach are discussed in section 4.1. Implementation of the proposed approach with real dataset and result analysis are shown in section 4.2.

\subsection{Theoretical analysis}

Computation complexity of any single participant in the proposed algorithm for phase-1 (set-up) is $\mathrm{O}(q)$ as required in the selection of random number $\left(r_{i} \in Z_{q}\right)$. Phase-2 (encrypt) completion is $\mathrm{O}(1)$ and phase-3 (sum) requires $\mathrm{O}(n)$ computations for the frequency count of any single itemset with $n$ participants. The overall computation complexity of proposed algorithm (i.e., set-up, encrypt and sum) becomes $\mathrm{O}(q)+\mathrm{O}(1)+\mathrm{O}(n)$. However, in real life situation the value of $q$ is much larger as compared with the number of involving parties, i.e., $n \ll q$. Thus, we can neglect the lower order terms and the final computation complexity will be $\mathrm{O}(q)$. Communication cost of each participant in phase-1 is $\mathrm{O}(1)$ as it requires sharing with its two neighbour participants in the ring as shown in figure 4 . Phase-2 (encrypt) requires the broadcast of the cipher text to $n-1$ participants. Hence, the communication cost of this phase is $\mathrm{O}(n)$. Last phase-3(sum) has communication cost of $\mathrm{O}(1)$, 


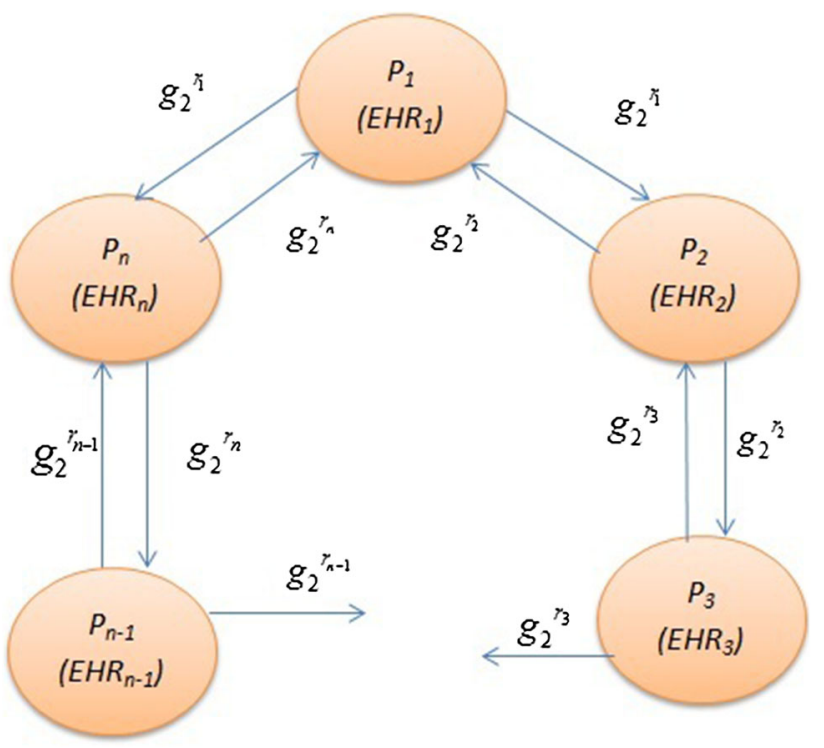

Figure 4. Phase-1 (Communication in initial set-up of participants.).
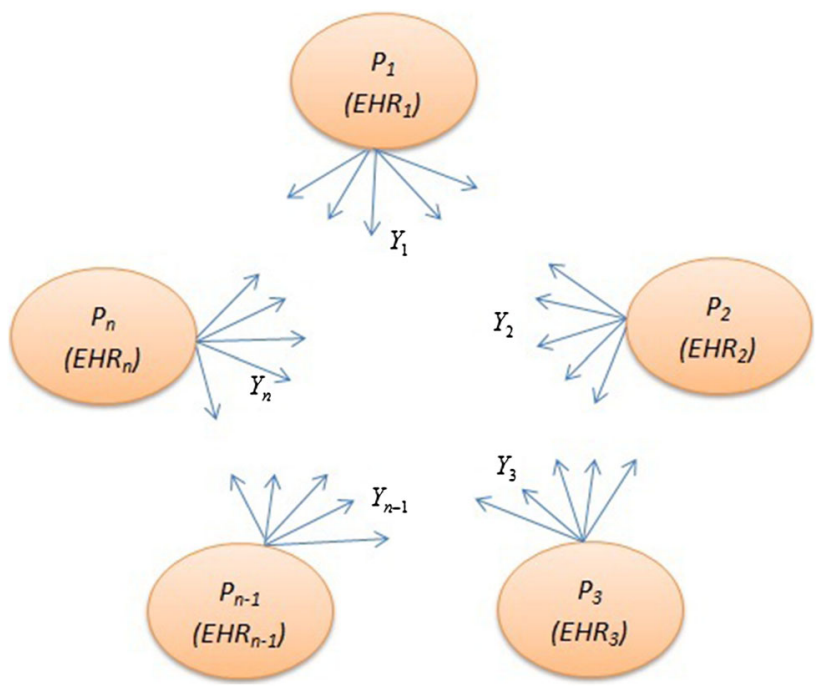

Figure 5. Phase-2 (communications of encrypted data).

as it does not require the communication with other participants. If one considers $N$ as a different candidate itemset in the dataset then phase- 2 and phase- 3 of proposed algorithm are executed $N$ times. Table 1 shows a summary of computation and communication complexity of each participants in the proposed approach and existing approach [22].

\subsection{Practical analysis}

In this section, implementation details and dataset used for evaluation are described. Practical result analysis of proposed approach is also presented. 4.2a Dataset: Publically available dataset of UCI heart diseases [11] is used to evaluate the proposed approach. The heart disease dataset has totally 76 different attributes with 303 patients in its record; 14 attributes that are linked with the heart disease are used. The details of these 14 attributes [39] are as follows:

1. Age: (numeric).

2. Sex: $(0=$ female, $1=$ male $)$.

3. Type of chest pain: $(1=$ typical angina, $2=$ atypical angina, $3=$ non-anginal pain, $4=$ asymptomatic).

4. Trestbps: (numeric), resting blood pressure.

5. Chol: (numeric), serum cholesterol in $\mathrm{mg} / \mathrm{dl}$.

6. Fasting blood sugar(fbs): $(1=(\mathrm{fbs}>120 \mathrm{mg} / \mathrm{dl})$ and 0 $=(\mathrm{fbs} \leq 120 \mathrm{mg} / \mathrm{dl}))$.

7. Resting electrocardiographic results (restecg): $(0=$ normal, $1=$ having ST-T wave abnormality ( $\mathrm{T}$ wave inversions and/or ST elevation or depression of $>0.05 \mathrm{mV}), 2=$ showing probable or definite left ventricular hypertrophy).

8. Thalach: (numeric), indicates the maximum heart rate achieved.

9. Exang: exercise-induced angina $(1=$ yes, $0=$ no $)$.

10. Oldpeak: (numeric), define ST depression induced by exercise relative to rest.

11. Slope: define the slope of the peak exercise ST segment( $1=$ upsloping, 2 = flat, $3=$ downsloping).

12. Ca : (numeric), number of major vessels $(0-3)$ coloured by fluoroscopy.

13. Thal: $(3=$ normal, $6=$ fixed defect, $7=$ reversible defect).

14. Class: diagnosis of the heart disease (angiographic disease status) $(0=$ healthy, $1=$ sick $)$.

4.2b Evaluation methodology: Four collaborative participants or EHR systems are used to evaluate the proposed approach. Therefore, the original dataset is randomly divided into four partitions. The results of each EHR system and the collaboration of four EHR systems are shown in table 2. Experiments are conducted using NetBeans on a machine with an intel core i3 CPU, 4 GB RAM, and $2.0 \mathrm{GHz}$ speed. Result of any single EHR system is compared to the result of the collaboration in terms of accuracy (confidence of the rule).

4.2c Result analysis: In terms of the result analysis, association rules with right hand side value class = healthy or sick are selected. Table 2 shows the details of association rules with confidence at each EHR system and collaborative EHR system.

The result shows that prediction of heart disease using any single EHR system has lower accuracy (confidence) in some EHR systems compared with the result of collaborative EHR systems. The analysis of experimental result based on heart disease dataset [11] shows that the female gender indicates lower chances of coronary heart disease. 
Table 1. Theoretical analysis of proposed approach and existing approach [22].

\begin{tabular}{|c|c|c|c|c|c|}
\hline \multicolumn{3}{|c|}{ Proposed approach } & \multicolumn{3}{|c|}{ Existing approach [22] } \\
\hline Per participant & $\begin{array}{l}\text { Computation } \\
\text { complexity }\end{array}$ & $\begin{array}{l}\text { Communication } \\
\text { complexity }\end{array}$ & Per participant & $\begin{array}{l}\text { Computation } \\
\text { complexity }\end{array}$ & $\begin{array}{l}\text { Communication } \\
\text { complexity }\end{array}$ \\
\hline $\begin{array}{l}\text { Phase-1( }\{\text { Set- } \\
\text { up }\})\end{array}$ & $\mathrm{O}(q)$ & $\mathrm{O}(1)$ & $\begin{array}{c}\text { Phase-1( }\{\text { Random polynomial } \\
\text { generation }\})\end{array}$ & $\mathrm{O}(q)$ & $\mathrm{O}(1)$ \\
\hline $\begin{array}{l}\text { Phase- } \\
2(\{\text { Encrypt }\})\end{array}$ & $\mathrm{O}(1)$ & $\mathrm{O}(n)$ & $\begin{array}{c}\text { Phase-2(\{Compute and share } \\
\text { the secrets }\})\end{array}$ & $\mathrm{O}(n)$ & $\mathrm{O}(n)$ \\
\hline Phase-3(\{Sum $\})$ & $\mathrm{O}(n)$ & $\mathrm{O}(1)$ & $\begin{array}{c}\text { Phase-3( } \begin{array}{c}\text { Compute sum of } \\
\text { secret values }\})\end{array}\end{array}$ & $\mathrm{O}(n)$ & $\mathrm{O}(n)$ \\
\hline Security model & \multicolumn{2}{|c|}{$\begin{array}{l}\text { Perfectly work with insecure } \\
\text { communication channel among semi- } \\
\text { honest participants and preserve the } \\
\text { privacy of each participant }\end{array}$} & Security model & \multicolumn{2}{|c|}{$\begin{array}{c}\text { Secure communication channel among } \\
\text { semi-honest participants is essential } \\
\text { to preserve the privacy of each } \\
\text { participant }\end{array}$} \\
\hline
\end{tabular}

Table 2. Result with confidence (\%) at each EHR system and proposed collaborative EHR system.

\begin{tabular}{|c|c|c|c|c|c|}
\hline \multirow[b]{2}{*}{ Association rules } & \multicolumn{5}{|c|}{ Confidence $(\%)$} \\
\hline & $\mathrm{EHR}_{1}$ & $\mathrm{EHR}_{2}$ & $\mathrm{EHR}_{3}$ & $\mathrm{EHR}_{4}$ & $\begin{array}{l}\text { Collaborative } \\
\text { EHR system }\end{array}$ \\
\hline $\begin{array}{l}\{\text { Sex }=\text { female }, \text { type of chest pain }=\text { asymptomatic, } \text { restecg }=\text { normal } \\
\text { exercise-induced angina }=\text { yes, slope }=\text { flat }\}=>\text { class sick }\end{array}$ & 90 & 85 & 86 & 92 & 99 \\
\hline $\begin{aligned}\{\text { Sex } & =\text { female }, \text { exercise-induced angina }=\text { no, number of vessels coloured }= \\
0\} & =>\text { class healthy }\end{aligned}$ & 92 & 88 & 85 & 83 & 98 \\
\hline $\begin{array}{l}\{\text { Type of chest pain }=\text { asymptomatic, } \text { slope }=\text { flat, thal }=\text { reversible } \\
\text { defect }\}=>\text { class sick }\end{array}$ & 89 & 88 & 89 & 93 & 96 \\
\hline $\begin{aligned}\{\text { Type of chest pain } & =\text { asymptomatic }, \text { exercise-induced angina }=\text { yes, thal }= \\
\text { reversible defect }\} & =>\text { class sick }\end{aligned}$ & 84 & 88 & 78 & 85 & 94 \\
\hline $\begin{array}{l}\{\text { Sex }=\text { male, } \text { slope }=\text { up, number of vessels coloured }=0, \text { thal }=\text { normal }\}= \\
\quad>\text { class healthy }\end{array}$ & 85 & 81 & 75 & 90 & 93 \\
\hline $\begin{array}{l}\{\text { Sex }=\text { male }, \text { type of chest pain }=\text { asymptomatic }, \text { restecg }=\text { hypertrophy } \\
\text { exercise-induced angina }=\text { yes }\}=>\text { class sick }\end{array}$ & 79 & 80 & 89 & 87 & 93 \\
\hline $\begin{array}{l}\{\text { Sex }=\text { male, type of chest pain }=\text { asymptomatic }, \text { fasting blood sugar }=\text { no, } \\
\text { exercise-induced angina }=\text { yes, thal }=\text { reversible defect }\}=>\text { class sick }\end{array}$ & 84 & 86 & 81 & 88 & 92 \\
\hline
\end{tabular}

Also, the result shows that the exercise-induced angina $=$ false is a sign of a person being healthy irrespective of the gender (major rules with high confidence contain exerciseinduced angina $=$ no in LHS part). Number of the coloured vessels $=0$ and thal $=$ normal are also signs of a healthy person. On the other hand, the rules of class 'sick' show that the thal $=$ reversed and type of chest pain = asymptomatic are probable signs of a person being sick (major high confidence rules have these two factors in LHS part).

Performance of distributed association rule mining algorithm depends on the number of collaborative participants and the $q$ (order of multiplicative group from which random number is selected). Proposed and existing approaches [22] are executed with different numbers of collaborative EHR systems by randomly dividing the data among them. We have evaluated the performance with $q=80$ bits security levels, as per NIST standard [40]. Computation time of the proposed approach and existing approach [22] with different numbers of collaborative EHR systems is shown in figure 6. Practical results show that the proposed approach is efficient compared with the existing approach [22] in terms of computation time and works in open communication channel. As shown in figure 6, computation time linearly increases with increasing total number of collaborative EHR systems. It shows that the proposed approach is scalable in terms of number of collaborative parties. 
Computation Time Vs Different Number of Collaborative EHR systems

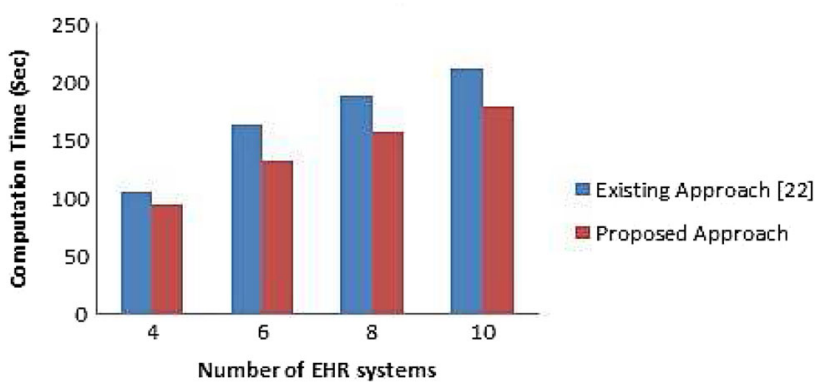

Figure 6. Performance comparison of proposed approach and existing approach [22] with different numbers of collaborative EHR systems.

\section{Conclusion}

In this work, we aimed to improve the accuracy of medical research by collaboration of multiple EHR systems with horizontally partitioned data. Privacy issues were discussed in the case of collaborating multiple EHR systems. To solve this, we proposed an efficient and secure algorithm for PPDARM on horizontally partitioned data. Experimental analysis of the proposed algorithm with heart disease data proved that the accuracy of heart disease prediction has improved and privacy is preserved. Further, proposed approach can be analysed for other diseases and can be extended for EHR systems with vertically partitioned data.

\section{Acknowledgements}

The authors are thankful to anonymous reviewers for their valuable suggestions to improve the quality of this paper.

\section{References}

[1] Tang P C and McDonald C J 2006 Electronic health record systems. Biomed. Inform. 10(4): 447-475

[2] Luukka P and Lampinen J 2010 A classification method based on principal component analysis and differential evolution algorithm applied for prediction diagnosis from clinical emr heart data sets. In: Computational intelligence in optimization: applications and implementations, vol. 7, Berlin-Heidelberg: Springer, pp. 263-283

[3] Ordonez C 2006 Association rule discovery with the train and test approach for heart disease prediction. IEEE Trans. Inf. Technol. Biomed. 10(2): 334-343

[4] Shin A M, Lee I H, Lee G H, Park H J, Park H S, Yoon K I, Lee J J and Kim Y N 2010 Diagnostic analysis of patients with essential hypertension using association rule mining. Healthc. Inform. Res. 16(2): 77-81

[5] Palaniappan S and Awang R 2013 Intelligent heart disease prediction system using data mining techniques. In:
Proceedings of the IEEE/ACS International Conference on Computer Systems and Applications pp. 108-115

[6] Clifton C, Kantarcioglu M and Vaidya J 2002 Defining privacy for data mining. In: Proceedings of the NSF workshop on Next Generation Data Mining 1(26): 199-204

[7] Gkoulalas-Divanis A, Loukides G and Sun J 2014 Publishing data from electronic health records while preserving privacy: a survey of algorithms. J. Biomed. Inform. 50: 4-19

[8] Australian Bureau of Statistics 2016 Causes of death in australia. [Online] Available: http://abs.gov.au/ausstats/abs. nsf/Products [accessed: 12-May-2016]

[9] Leading-causes-of-death, australia. [Online] Available: http://www.aihw.gov.au/deaths/leading-causes-of-death [accessed: 12-May-2016]

[10] Gulbinat W The role of WHO as an intergovernmental organization in the coordination of telematics in health care. [Online] Available: https://www.hon.ch/Library/papers/gul binat.html [accessed: 19-April-2016]

[11] Heart disease dataset. [Online] Available: http://archive.ics. uci.edu/ml/machine-learningdatabases/heart-disease/cleve. mod [accessed: 28-May-2016]

[12] Definition of heart disease. [Online] Available: http://www. healthscout.com/ency/68/458/main.html [accessed: 12-March-2016]

[13] Andersen L B and Haraldsdttir J 1993 Tracking of cardiovascular disease risk factors including maximal oxygen uptake and physical activity from late teenage to adulthood an 8-year follow-up study. J. Intern. Med. 234(3): 309-315

[14] Kantarcioglu M and Clifton C 2004 Privacy preserving distributed mining of association rules on horizontally partitioned data. IEEE Trans. Knowl. Data Eng. 16(9): 1026-1037

[15] Kantarcioglu M 2008 A survey of privacy-preserving methods across horizontally partitioned data. In: Proceedings of privacy preserving data mining of advances in database systems 34, pp. 313-335

[16] Ge X, Yan L, Zhu J and Shi W 2010 Privacy preserving distributed association rule mining based on the secret sharing technique. In: Proceedings of the 2nd International Conference on Software Engineering and Data Mining (SEDM), pp. 345-350

[17] Verykios V S and Gkoulalas-Divanis A 2008 A survey of association rule hiding methods for privacy. Proceedings of privacy preserving data mining 34: 267-289

[18] Lin C W, Hong T P and Hsu H C 2014 Reducing side effects of hiding sensitive itemsets in privacy preserving data mining. Sci. World J. 2014: 267-289

[19] Malik M B, Ghazi M A and Ali R 2012 Privacy preserving data mining techniques: current scenario and future prospects. In: Proceedings of the Third International Conference on Computer and Communication Technology (ICCCT) pp. 26-32

[20] Aggarwal C C and Philip S Y 2008 A general survey of privacy-preserving data mining models and algorithms. In: Privacy preserving data mining pp. 11-52

[21] Lindell Y and Pinkas B 2000 Privacy preserving data mining. In: Proceedings of CRYPTO 2000, Lecture Notes in Computer Science, vol. 1880 , pp. 36-54

[22] Nanavati N R, Lalwani P and Jinwala D C 2014 Analysis and evaluation of schemes for secure sum in collaborative 
frequent itemset mining across horizontally partitioned data. J. Eng. 2014: 110-120

[23] Nguyen X C, Le H B and Cao T A 2014 An enhanced scheme for privacy-preserving association rules mining on horizontally distributed databases. In: Proceedings of the IEEE International Conference on Computing and Communication Technologies, Research, Innovation, and Vision for the Future (RIVF), pp. 1-4

[24] Jayalakshmi S and Moses T J 2014 Privacy preserving mining of association rules in horizontally distributed databases. Int. J. Manage. IT Eng. 4(9): 209-222

[25] Ouda M A, Salem S A, Ali I A and Saad E S M 2012 Privacy preserving data mining (PPDM) method for horizontally partitioned data. Int. J. Comput. Sci. Iss. 9(5): 339-347

[26] Vaidya J S 2004 Privacy preserving data mining over vertically partitioned $d$ Data. PhD Dissertation, Purdue University, USA

[27] Kargupta H, Das K and Liu K 2007 Multi party, privacy preserving distributed data mining using a game theoretic framework. In: Proceedings of the European Conference on Principles of Data Mining and Knowledge Discovery, pp. 523-531

[28] Nanavati N R and Jinwala D C 2015 A novel privacy preserving scheme for collaborative frequent itemset mining across vertically partitioned data. Sec. Commun. Netw. 8(18): 4407-4420

[29] Xu Z and Yi X 2011 Classification of privacy-preserving distributed data mining protocols. In: Proceedings of the Sixth International Conference on Digital Information Management, pp. 337-342

[30] Alwatban I S and Emam A Z 2014 Comprehensive survey on privacy preserving association rule mining: models, approaches, techniques and algorithms. Int. J. Artif. Intell. Tools 23(05): 145-162
[31] Zhu X M 2013 Research on privacy preserving data mining association rules protocol. Adv. Mater. Res. 756: $1661-1664$

[32] Agrawal R and Srikant R 1994 Fast algorithms for mining association rules. In: Proceedings of the 20th International Conference on Very Large Data Bases, VLDB, vol. 1215, pp. 487-499

[33] Sweeney L 2013 Matching known patients to health records in washington state data. Technical Report, Data Privacy Lab

[34] Karabatak M and Ince M C 2009 An expert system for detection of breast cancer based on association rules and neural network. Expert Syst. Appl. 36(2): 3465-3469

[35] Kou G, Peng Y, Shi Y and Chen Z 2007 Privacy preserving data mining of medical data using data separation based techniques. Data Sci. J. 6: S429-S434

[36] Lindell Y and Pinkas B 2009 Secure multiparty computation for privacy-preserving data mining. J. Privacy Confid. 1(1): 59-98

[37] Joux A and Nguyen K 2003 Separating decision DiffieHellman from computational Diffie-Hellman in cryptographic groups. J. Cryptol. 16(4): 239-247

[38] Jung T, Li X Y and Wan M 2015 Collusion tolerable privacy preserving sum and product calculation without secure channel. IEEE Trans. Dependable Secure Comput. 12(1): 45-57

[39] Cleveland heart disease data details. [Online] Available: http://archive.ics.uci.edu/ml/machine-learning-databases/ heart-disease/heart-disease.names [accessed: 28-May-2016]

[40] Barker E, Barker W, Burr W, Polk W, Smid M and Gallagher P D 2007 NIST special publication 800-57, recommendation for key management part 1: general. NIST Spec. Pub. 800(57), pp. 1-147 